\section{Thromboserisiko bei RA-Patienten}

Molander $\mathrm{V}$ et al. Does the risk of venous thromboembolism vary with disease activity in rheumatoid arthritis?. doi:10.1136/annrheumdis-2020-eular.353

Patienten mit rheumatoider Arthritis (RA) und hoher Krankheitsaktivität leiden besonders häufig unter Thrombosen. Eine aktuelle schwedische Studie kommt zu dem Ergebnis, dass unter $\mathrm{Pa}$ tienten mit hoher Krankheitsaktivität einer von hundert innerhalb eines Jahres eine venöse Thromboembolie entwickelt - das entspricht einem Anstieg um mehr als das Zweifache im Vergleich zu Patienten in Remission.

Eine Therapie mit sogenannten biological disease-modifying antirheumatic drugs (bDMARD) kann dieses erhöhte ThromboseRisiko reduzieren, zeigen Daten des deutschen RABBIT ${ }^{1}$-Registers, die im Vorfeld des Europäischen Rheumatologenkongresses EULAR (European League against Rheumatism, EULAR) veröffentlicht wurden. Diese Information ist besonders angesichts der aktuellen Pandemie von Bedeutung, denn auch bei COVID-19 spielen Thrombosen und Lungenembolien eine große Rolle im Erkrankungsgeschehen. Weltweit untersuchen Wissenschaftler, wie sich eine SARS-CoV2-Infektion auf Patienten mit entzündlichrheumatischen Erkrankungen auswirkt, um daraus Handlungsempfehlungen für die Therapie abzuleiten. EULAR rät, bei der Behandlung von Patientinnen und Patienten mit Rheuma immer an das Thromboserisiko zu denken und die Krankheitsaktivität regelmäßig zu kontrollieren.

Welche Faktoren das Entstehen einer Thrombose bei Patientinnen und Patienten mit RA begünstigen und welche Medikamente das Risiko reduzieren könnten, wurde jetzt in 2 aktuellen Studien untersucht. Eine schwedische Kohorten-Studie ging der Frage nach, ob der Grad der Krankheitsaktivität das Thrombose-Risiko beeinflusst. Das Team um Viktor Molander, Doktorand am Karolinska Institutet in Stockholm, hat die Daten von 46311 Patientinnen und Patienten mit RA aus dem schwedischen Qualitätsregister für Rheumatologie (SRQ) über einen Zeit- raum von 12 Jahren analysiert. Für die Messung der Krankheitsaktivität wurde der „Disease Activity Score“ (DAS) verwendet. Mit dem DAS28 wird die Krankheitsaktivität der rheumatoiden Arthritis auf der Basis von 28 definierten Gelenken erfasst.

Die Studie belegt einen starken Zusammenhang zwischen der mit dem DAS28 gemessenen Krankheitsaktivität der RA und dem Risiko einer VTE: „Unter den Patienten mit hohen Entzündungswerten wird einer von hundert innerhalb des kommenden Jahres eine VTE entwickeln - das entspricht einer mehr als 2-fachen Zunahme im Vergleich zu Patienten in Remission“, fasst Molander die Ergebnisse zusammen.

„Die regelmäßige Untersuchung und Prüfung der Krankheitsaktivität beim Rheumatologen kann lästig sein, ist jedoch sehr wichtig, um zu überprüfen, wie sich die Krankheit entwickelt und ob die Behandlung entsprechend angepasst werden muss“, so Professor Dr. med. John Isaacs, The University of Newcastle, Großbritannien, Vorsitzender des wissenschaftlichen ProgrammKomitees beim EULAR.

\section{Biologika können Thromboserisiko reduzieren}

Auch die Medikation bei Rheuma-Erkrankungen hat Einfluss auf das Thrombose-Risiko. Sogenannte conventional synthetic disease-modifying antirheumatic drugs (csDMARD) wie Methotrexat, Sulfasalazin und Leflunomid gehören zur Basistherapie bei RA. Eine Alternative bilden die Biologika (bDMARD). Ob der Einsatz von bDMARDs wie TNF-Inhibitoren im Vergleich zu csDMARDs das Thrombose-Risiko reduziert, haben Wissenschaftler um den Erstautor Dr. rer. nat. Martin Schäfer vom Programmbereich Epidemiologie des Deutschen Rheuma-Forschungszentrums in Berlin untersucht. Hierfür hat das Team die Daten von mehr als 11000 RA-Patientinnen und -Patienten aus dem deutschen RABBIT-Register analysiert, die nach mindestens einem csDMARD-Versagen entweder mit einem weiteren csDMARD behandelt oder auf die Therapie mit einem bDMARD umgestellt wurden.

Das Ergebnis: „Die Behandlung mit TNFHemmern reduzierte das Risiko schwerer
VTE-Ereignisse im Vergleich zu csDMARDs um fast die Hälfte“, erklärt Schäfer. Eine erhöhte Entzündungsaktivität war auch anhand der RABBIT-Daten mit einem signifikant erhöhten Risiko für VTEs assoziiert: Ein CRP-Wert von mindestens $5 \mathrm{mg} /$ l verdoppelte das Risiko annähernd. „Aus den Ergebnissen können wir jedoch nicht ableiten, dass Patienten mit entzündlichen rheumatologischen Erkrankungen durch ihre Therapie mit TNF-Hemmern vor einem schweren Verlauf einer COVID-19-Infektion geschützt sind“, so Isaacs. „Vielmehr warnen wir ausdrücklich davor, Biologika ohne medizinische Indikation zum Schutz vor einer SARS-CoV-2-Infektion oder einem schweren Verlauf einer COVID-19-Erkrankung einzunehmen“.

Nach einer Pressemitteilung des Europäischen Rheumatologenkongresses. 